\title{
Maîtriser avec succès le retour à la profession
}

Marianna Bodenmann-Zanetti ${ }^{a}$, Judith Naef ${ }^{b}$

a Dr méd., membre du comité; ${ }^{b}$ avocate, directrice de «mws - medical women switzerland»

Le projet «Steigbügel» de medical women switzerland et des groupes Medbase vise à encourager le retour à la profession dans la médecine de premier recours. L'ISFM, la FMF et la SSMIG soutiennent le projet. Il s'adresse aux médecins hommes et femmes qui, après une pause consacrée à la famille, souhaitent terminer leur formation postgraduée pour obtenir le titre en médecine interne générale ou faire leurs premières expériences après l'obtention de ce titre de spécialiste.

Pendant des années, les universités suisses n'ont pas formé suffisamment de médecins. Par ailleurs, d'après une étude récemment publiée par la FMH et l'ASMAC, environ 10 pourcent de ces médecins n'exercent plus d'activité auprès des patients après quelques années seulement. Selon un sondage de l'association femmes médecins suisse «mws - medical women switzerland», ce sont notamment les femmes médecins qui veulent reprendre leur profession pour se consacrer aux patients. De plus, elles souhaitent bénéficier d'un conseil de carrière.

\section{Expertes expérimentées et coaching orienté selon les besoins}

mws répond à ces attentes par son projet novateur «Steigbügel»: il s'adresse à des femmes médecins qui

\section{Avis de recherche!}

Nous cherchons: médecin, femme ou homme, ayant (presque) terminé sa formation en médecine interne générale, actuellement en congé parental et désireux de reprendre une activité dans la médecine de premier recours. Les personnes intéressées sont priées de s'annoncer chez Marianna Bodenmann-Zanetti, spécialiste en médecine interne, praticienne formatrice passionnée, mère de trois enfants adultes et responsable du projet «Steigbügel»: Dr méd. Marianna Bodenmann-Zanetti, Eichstr. 4, 8620 Wetzikon, courriel: marianna.bodenmann[at]medbase.ch ou marianna.bodenmann[at] medicalwomen.ch. Elle se réjouit de répondre à votre demande!

Vous trouverez d'autres informations sur www.medicalwomen.ch et www. medbase.ch. sont sur le point de terminer leur formation postgraduée en médecine interne générale ou qui souhaitent faire leurs premières expériences en médecine de premier recours après l'obtention de ce titre de spécialiste. Les futures médecins de premier recours, qui n'ont pas exercé leur profession pendant au moins douze mois pour des raisons familiales, peuvent, grâce au partenaire de projet Medbase, poursuivre leur formation postgraduée jusqu'à l'obtention du titre de spécialiste. Leur formation de spécialiste presque terminée, elles travaillent pendant une année dans un cabinet Medbase en tant que médecins-assistantes à 50 pourcent et peuvent ainsi valider six mois d'assistanat pour la formation postgraduée. Le retour à la profession est soutenu par un coaching individuel et de groupe spécialement adapté aux besoins de ce groupe et par des praticiennes et praticiens enseignants formés à cet effet. Bien évidemment, d'autres cabinets de groupe ou individuels extérieurs au groupe Medbase sont les bienvenus dans le projet. Ceux-ci disposeront de préférence d'une solide expérience dans l'assistanat au cabinet et de femmes médecins susceptibles de participer au programme. mws informera les offices cantonaux qui financent le programme d'assistanat en cabinet déjà établi du programme et de ses spécificités. La question de savoir s'ils prendront en charge les coûts salariaux des assistants au cabinet dans la mesure usuelle reste toutefois ouverte. La participation au programme pourra donc générer des coûts importants pour les cabinets formateurs. D'un autre côté, ces derniers contribueront ainsi à assurer l'avenir du système de santé et pourront éventuellement fidéliser de futurs collaborateurs. 


\section{Un retour progressif à la profession}

L'assistanat au cabinet dans le cadre du projet «Steigbügel» ne se distingue donc des autres programmes d'assistanat au cabinet payés ni sur le plan du contenu, ni sur les objectifs, mais seulement dans son approche: l'expérience montre qu'après une pause pour raisons familiales de seulement deux ou trois ans déjà, un effort particulier et un soutien spécifique sont souvent nécessaires pour reprendre pied avec succès dans la profession. Il faut rétablir une certaine routine et assurance pour réussir son retour à la profession. De nos jours, le succès n'est souvent pas au rendez-vous, car les exigences auxquelles les assistantes en cabinet doivent satisfaire après une pause familiale sont vite trop élevées. Souvent, on attend des assistantes que dès le premier jour de travail, elles soient en mesure de faire appel à tout leur savoir et à ne plus penser aux questions privées. On ne leur donne pas la possibilité de se réadapter au monde du travail et d'apprendre, en plus de cela, à organiser et gérer le quotidien familial. Il est sans aucun doute plus facile de surmonter ce grand défi si toutes les parties prenantes tiennent compte de ces circonstances particulières, sans toutefois perdre de vue l'objectif: après l'année effectuée dans le cadre du projet "Steigbügel», les médecins doivent être solidement réinstallées dans leur profession pour s'engager avec résolution dans la médecine de premier recours.

\section{Démarrage du projet en mars 2018}

Le projet «Steigbügel» est réparti sur trois ans de mars 2018 à février 2020. Il sera réalisé sur cinq cycles avec chaque fois au maximum huit participantes - les participants sont aussi les bienvenus, s'ils satisfont aux critères. Pendant douze mois dans le cadre d'une formation «1:1»-on-the-job, les participants sont accompagnés de praticiennes et de praticiens formateurs spécialement formés. En gagnant de l'expérience et de l'assurance, les participantes et participants deviennent de plus en plus autonomes dans leur travail, comme dans le programme d'assistanat au cabinet de la Fondation pour la promotion de la formation en médecine de famille (FMF). Ils suivent leurs formations et manifestations ainsi que les cercles de qualité et formations continues internes au cabinet. De plus, le retour à la profession est encadré par le coaching spécifique individuel et de groupe.

\section{Un large soutien}

Outre le partenaire de projet Medbase, l'Institut suisse pour la formation médicale postgraduée et continue (ISFM), la Société suisse de médecine interne générale (SSMIG) et la Fondation pour la promotion de la formation en médecine de famille (FMF) soutiennent ce projet pilote. L'Institut d'enseignement médical (IML) de la faculté de médecine de l'Université de Berne procédera à l'évaluation pour identifier les facteurs de succès et élaborer des recommandations concrètes, afin que les expériences faites dans le cadre du projet «Steigbügel» puissent aussi bénéficier à d'autres disciplines, régions linguistiques et entreprises. Le projet est soutenu par le Secrétariat d'Etat à l'économie (SECO) dans le cadre de l'initiative visant à combattre la pénurie de personnel qualifié et par le Bureau fédéral de l'égalité entre femmes et hommes (BFEG) avec des aides financières conformément à la loi sur l'égalité.

Le projet «Steigbügel» vise par ailleurs à inciter les médecins, hommes et femmes, qui ont cessé leur activité auprès des patients à reprendre une activité dans l'intérêt du système de santé.

Le projet «Steigbügel» vise par ailleurs à inciter les médecins, hommes et femmes, qui ont cessé leur activité auprès des patients à reprendre une activité dans l'intérêt du système de santé. Il ne se focalise donc pas seulement sur le soutien direct des participantes et participants, mais aussi sur la sensibilisation et la formation spécifique des praticiennes et praticiens formateurs. Bien sûr, il est soumis à une évaluation scientifique avec pour objectif de rendre les expériences accessibles à tous et d'encourager le retour à la profession des médecins dans d'autres disciplines.

\section{Un bénéfice économique}

Du point de vue économique aussi, le retour à la profession des médecins doit impérativement être encouragé: la société investit environ 1 million de francs dans chaque médecin pour les plus de dix ans de formation pré- et postgraduée jusqu'au titre de spécialiste. En comparaison, les coûts de 40000 francs pour le coaching et les praticiens formateurs sont relativement faibles. 